\title{
Divalent-doped $\mathrm{Na}_{3} \mathrm{Zr}_{2} \mathrm{Si}_{2} \mathrm{PO}_{12}$ Natrium Superionic Conductor: Improving the ionic conductivity via simultaneously optimizing the phase and chemistry of the primary and secondary phases
}

Mojtaba Samiee ${ }^{\dagger}$, Balachandran Radhakrishnan ${ }^{\dagger}$, Zane Rice, Zhi Deng, Ying Shirley Meng, Shyue Ping Ong ${ }^{*}$, Jian Luo*

Department of NanoEngineering, University of California San Diego, 9500 Gilman Drive, Mail Code 0448, La Jolla, CA 92093, USA

E-mail: jluo@alum.mit.edu (J.L.), ongsp@eng.ucsd.edu (S.P.O.)

Keywords: NASICON, Doping, Solid-State Electrolyte, Grain Boundary Conductivity, Sodium Superionic Conductor.

\section{Abstract}

NASICON is one of the most promising sodium solid electrolytes that can enable the assembly of cheaper and safer sodium all-solid-state batteries. In this study, we perform a combined experimental and computational investigation into the effects of aliovalent doping in NASICON on both bulk and grain boundary (secondary phase) ionic conductivity. Our results show that the dopants with low solid solubility limits in NASICON lead to the formation of a conducting (less insulating) secondary phase, thereby improving the grain boundary conductivity measured by electrochemical impedance spectroscopy (including grain-boundary, secondary-phase, and other microstructural contributions) that is otherwise hindered by the poorly-conducting secondary phases in undoped NASICON. This is accompanied by a change in the $\mathrm{Si} / \mathrm{P}$ ratio in the primary NASICON bulk phase, thereby transforming monoclinic NASICON to rhombohedral NASICON. Consequently, we have synthesized NASICON chemistries with significantly improved and optimized total ionic conductivity of $2.7 \mathrm{mS} / \mathrm{cm}$. More importantly, this study has achieved a understanding of the underlying mechanisms of improved conductivities via doping (differing from the common wisdom) and further suggests a new general direction to improve the ionic conductivity of

\footnotetext{
${ }^{\dagger}$ M.S. and B.R. contributed equally to this work.

*Correspondence should be addressed to: J.L. (jluo@alum.mit.edu) and S.P.O. (ongsp@eng.ucsd.edu).
} 
solid electrolytes via simultaneously optimizing the primary bulk phase and the microstructure (including grain boundary segregation and secondary phases).

\section{Introduction}

The development of all-solid-state rechargeable alkali-ion batteries is an important step towards safer, higher energy density storage for large-scale applications such as automotive and grid storage. A key component in such an energy storage device will be the solid-state electrolytes with extremely high alkali ionic conductivities, also known as alkali superionic conductors, with room temperature conductivities in excess of $1 \mathrm{mS} / \mathrm{cm}$ being a typical target.

Among the known superionic conductors, the NAtrium SuperIonic CONductor (NASICON) family of compounds, with general formula $\mathrm{Na}_{1+x} \mathrm{Zr}_{2} \mathrm{Si}_{x} \mathrm{P}_{3-x} \mathrm{O}_{12}(0 \leq x \leq 3$, and $\mathrm{Zr}$ can be substituted with other metals or mixture of metals), which has first been developed by Hong and Goodenough in 1976 [1,2], are arguably one of the most well-known and studied. Originally conceived as a replacement for $\beta$-alumina in high-temperature Na-S batteries, NASICON-based compounds have nevertheless garnered significant interest for low/roomtemperature applications as electrodes and solid electrolytes for both $\mathrm{Li}$ and Na-ion rechargeable batteries [3-6]. Unlike sulfide solid electrolytes [7-11], NASICONs are oxides, which are considerably more air and moisture stable [12-15]. However, the major challenge for NASICONs thus far are their typically moderate-to-low total (bulk plus grain boundary) ionic conductivities at room temperature $(\sim 0.1 \mathrm{mS} / \mathrm{cm})$ due to the poorly-conducting $\mathrm{ZrO}_{2}$ phases formed at grain boundaries [16-20]. Tetragonal $\mathrm{Na}_{3} \mathrm{PO}_{4}$ is another phase formed at grain boundaries of NASICONs with different synthesis procedures that has received much less attention in literature $[4,13]$. It should be noted that the so-called "grain boundary conductivity" (measured by electrochemical impedance spectroscopy) in fact represents the 
combination of the (often coupled) contributions from grain boundaries, secondary phases, and other microstructural features (e.g., the size and distribution of the primary and secondary phases, along with any interfacial effects at the hetero-phase boundaries). In fact, this current study focused more on the effects of secondary phases (precipitated at grain boundaries) than the actual grain boundaries themselves, although we recognize that the latter, the actual grain boundaries (including grain boundary adsorption/segregation and possible formation of nanoscale interfacial phases at grain boundaries; see, e.g., a recent review [51] for elaboration), can also make significant contributions to a blocking effect.

There are two known phases of NASICONs - a rhombohedral R $\overline{3} c$ phase and a monoclinic $\mathrm{C} 2 / \mathrm{c}$ phase that is formed, for example, by $\mathrm{Na}_{1+x} \mathrm{Zr}_{2} \mathrm{Si}_{x} \mathrm{P}_{3-x} \mathrm{O}_{12}$ for $1.8 \leq x \leq 2.2$ at temperatures below $160^{\circ} \mathrm{C}$ - that are related to each other by a small rotational distortion. Fig. 1 shows the rhombohedral and monoclinic crystal structures, which comprises corner-sharing $\mathrm{SiO}_{4} / \mathrm{PO}_{4}$ tetrahedra and $\mathrm{ZrO}_{6}$ octahedra. In the rhombohedral form, two distinct $\mathrm{Na}$ sites $\mathrm{M}_{1}$ and $\mathrm{M}_{2}$ form a 3D diffusion network, while the monoclinic distortion splits the $\mathrm{M}_{2}$ sites into $M_{2}^{\alpha}$ and $M_{2}^{\beta}$ sites.

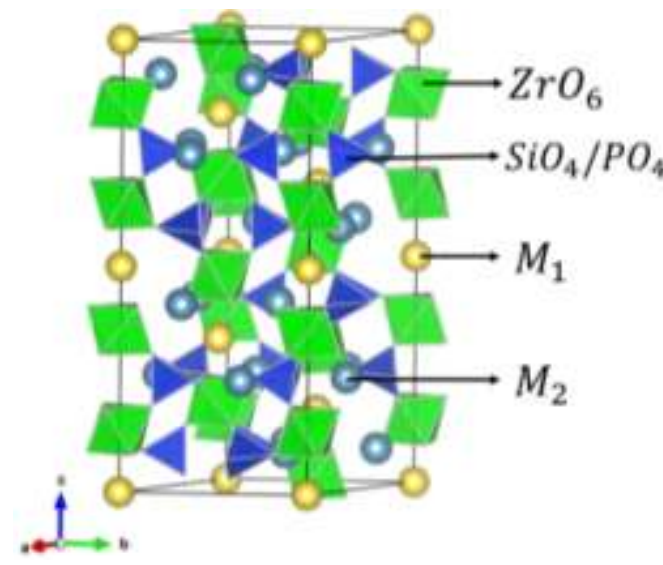

(a)

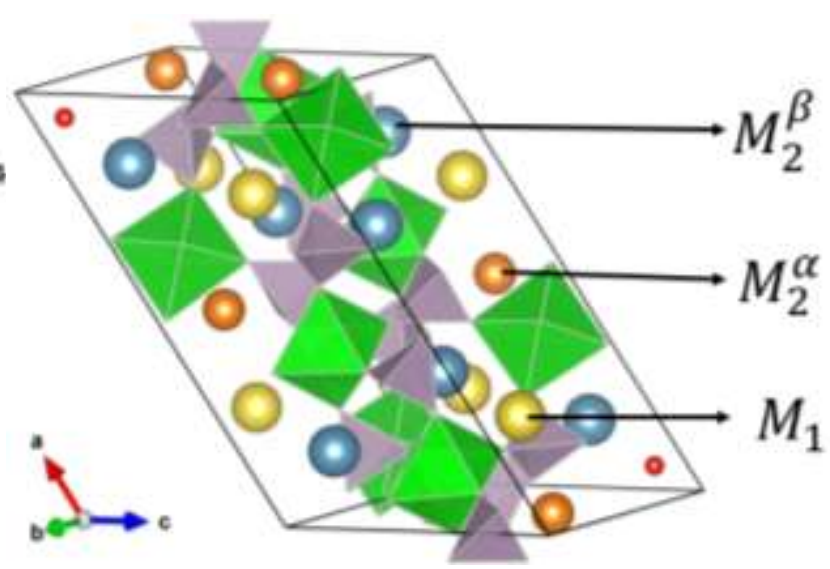

(b)

Fig. 1. Conventional unit cells of NASICON. (a) Rhombohedral structure (b) Monoclinic structure. The rhombohedral structure has two distinct $\mathrm{Na}^{+}$sites: $\mathrm{M}_{1}$ and $\mathrm{M}_{2}$. The local $\mathrm{Si} / \mathrm{P}$ 
environment and the monoclinic distortion splits the $\mathrm{M}_{2}$ sites into two different sites: $M_{2}^{\alpha}$ and $M_{2}^{\beta}$ labeled in the figure.

Recently, there have been several efforts aimed at improving the ionic conductivity of NASICONs by aliovalent doping $[4,5,21]$. Jolley et al. studied the effect of aliovalent dopants $\left(\mathrm{Ni}^{2+}, \mathrm{Zn}^{2+}, \mathrm{Al}^{3+}, \mathrm{Fe}^{3+}, \mathrm{Y}^{3+}\right)$ on the ionic conductivity of NASICON. Although all dopants provided room temperature conductivities higher than undoped NASICON, only $\mathrm{Co}^{2+}$ doped NASICON provided a conductivity of higher than $1 \mathrm{mS} / \mathrm{cm}(1.55 \mathrm{mS} / \mathrm{cm})$ at room temperature [4]. However, a Co-based NASICON is unlikely to be a good solid electrolyte candidate due to the redox active nature of Co metal. More recently, Ma et al. synthesized $\mathrm{Sc}^{3+}$-doped NASICON that achieved an overall ionic conductivity of $4.0 \mathrm{mS} / \mathrm{cm}$ at room temperature, which is very similar to the value $(3.7 \mathrm{mS} / \mathrm{cm})$ obtained in a proprietary compound NASICON reported by Ceramatec [5,22], although Sc is neither an earth-abundant nor a cost-effective doping element. Ma et al. attributed the improvement in bulk conductivity to a higher $\mathrm{Na}$ concentration in doped NASCION because substitution of $\mathrm{Zr}^{4+}$ ions with $\mathrm{M}^{3+} / \mathrm{M}^{2+}$ ions, and the enhancement in grain boundary conductivity to the improved microstructure from the solution-assisted solid-state synthesis procedure. However, the improvement in grain boundary conductivity can also be inferred from normalized Nyquist plots of aliovalent-doped NASICON synthesized by solid-state reaction method [4].

In this work, we present a comprehensive experimental and first principles study of the effect of earth-abundant divalent dopants such as $\mathrm{Mg}^{2+}$ and $\mathrm{Ni}^{2+}$ on the ionic conductivity of NASICON $\mathrm{Na}_{1+\mathrm{x}} \mathrm{Zr}_{2} \mathrm{Si}_{\mathrm{x}} \mathrm{P}_{3-\mathrm{x}} \mathrm{O}_{12}$, with an emphasis on understanding the underlying mechanisms of conductivity improvements, particularly the interplays between doping, solubility, segregation, and formation of secondary phases. Unlike previously held 
assumptions, we will demonstrate conclusively that the NASICON structure has in fact limited solubility for divalent dopants, which will in fact mostly dissolve into the secondary precipitation phases at grain boundaries, such as $\mathrm{Na}_{3} \mathrm{PO}_{4}$. We show that instead, divalent dopants modify the primary-secondary phase equilibria by modifying the $\mathrm{Si} / \mathrm{P}$ ratio (and consequently, the $\mathrm{Na}$ concentration and space group) in the bulk and creating diffusionassisting defects in the secondary phase. A maximum conductivity of $2.7( \pm 0.3) \mathrm{mS} / \mathrm{cm}$ was achieved with a Mg-doped NASICON with $\mathrm{Si} / \mathrm{P}$ ratio of 2.2 which should be a viable candidate solid electrolyte for room-temperature rechargeable all-solid-state sodium-ion batteries. These insights pave the way for further composition tuning of earth-abundant NASICON compounds. Moreover, this study demonstrated the importance of simultaneous optimizing the primary (bulk) and secondary phases (plus the microstructure and interfaces) to achieve better overall performance, guided by the modeling of both phase formation and conductivity (vs. an overly-simplified assessment of the "doping" effect on conductivity via considering only the consequence of substituting cations in the primary bulk phase), which represents a strategy that should be generally adopted to improve other solid electrolytes.

\section{Experimental Methods}

\subsection{Synthesis}

NASICON specimens with a nominal composition of $\mathrm{Na}_{3+2 x} \mathrm{Zr}_{2-x} M_{x} \mathrm{Si}_{2} \mathrm{PO}_{12}\left(M=\mathrm{Mg}^{2+}, \mathrm{Ni}^{2+}\right)$ were prepared by a solid-state procedure using $\mathrm{Na}_{2} \mathrm{CO}_{3}$ (Fisher Scientific, $99.5 \%$ ), $\mathrm{ZrO}_{2}$ (Fisher Scientific, laboratory grade), $\mathrm{SiO}_{2}$ (Alfa Aesar, 99.9\%), $\mathrm{NH}_{4} \mathrm{H}_{2} \mathrm{PO}_{4}$ (Sigma Aldrich, 99.99\%), and $\mathrm{MgO}$ (Alfa Aesar, 99.95\%) or NiO (Alfa Aesar, 99.998\%) as precursors. The precursors were mixed using planetary ball mill in isopropanol for $24 \mathrm{~h}$ and then dried in an oven at $85^{\circ} \mathrm{C}$ overnight. Calcination was performed at $1150{ }^{\circ} \mathrm{C}$ for $5 \mathrm{~h}$ with a heating rate of $5{ }^{\circ} \mathrm{C} / \mathrm{min}$ in air. The calcined powders were manually ground using a pestle and mortar, followed by further grounding in a planetary ball mill in isopropanol for $48 \mathrm{~h}$. The NASICON 
powders were then pressed using uniaxial pressing at about 2 metric tons (at a pressure of $\sim 62$ $\mathrm{MPa}$ ) to form pellets of about $20 \mathrm{~mm}$ diameter and 3-4 $\mathrm{mm}$ thickness. Sintering was performed at $1230{ }^{\circ} \mathrm{C}$ and $1300{ }^{\circ} \mathrm{C}$ for $24 \mathrm{~h}$ with a heating rate of $5^{\circ} \mathrm{C} / \mathrm{min}$ for $\mathrm{Na}_{3} \mathrm{Zr}_{2} \mathrm{Si}_{2} \mathrm{PO}_{12}$ and $\mathrm{Na}_{3.2} \mathrm{Zr}_{2} \mathrm{Si}_{2.2} \mathrm{PO}_{12}$ series specimens, respectively. Extra powders of the same composition were placed both underneath and on the top of the pellets (so that the pellets were buried in the powders of identical composition) to (1) avoid the contamination from boat and (2) to reduce the evaporation. After sintering, both sides of pellets were polished to remove $0.5-1$ $\mathrm{mm}$ material from top and bottom of each pellet to further ensure that the homogeneous parts of the pellets were used for conductivity measurements and other characterization. The relative densities of $>90 \%$ were achieved for all sintered specimens with different compositions.

\subsection{Characterization}

X-ray diffraction (XRD) measurements of the specimens were performed using $\mathrm{Cu} \mathrm{K}_{\alpha}$ radiation to identify the crystalline phases. The microstructures of the cross sections of sintered pellets were observed using Scanning Electron Microscopy (SEM, FEI XL30) equipped with an energy-dispersive spectroscopy (EDS) analysis system (iXRF).

\subsection{Conductivity measurement}

Na-ion conductivity was measured with electrochemical impedance spectroscopy (EIS) using an impedance analyzer (Solartron 1255B) in the frequency range of $1 \mathrm{MHz}$ to $1 \mathrm{~Hz}$ at 25, 0, 20, $-40,-60$ and $-80{ }^{\circ} \mathrm{C}$ with Pt blocking electrodes sputtered on both sides of the pellets inside a temperature test chamber (LR Environmental Equipment Company) in dry air. Grain boundary, bulk and total conductivity were determined by fitting Nyquist plots (Fig. S1 and S2 in the Supplementary Information) using the Z-View software (Scribner, Inc.). Activation 
energies for specific grain boundaries, bulk and total conductivities were determined from fitting Arrhenius plots (Fig. S3 and S4 in the Supplementary Information).

\section{Computational methods}

\subsection{Density functional theory calculations}

All density functional theory (DFT) calculations were performed using Vienna Ab-initio Simulations Package (VASP) [23] within the Projector Augmented Wave (PAW) method. The Perdew-Burke-Ernzerhof (PBE) generalized gradient approximation was used to model the exchange-correlation functional. A plane-wave cut-off of $520 \mathrm{meV}$ was applied with a $k$ point mesh $4 \times 4 \times 2$. An electronic energy tolerance of $5 \times 10^{-5} \mathrm{eV} /$ atom was used to relax the crystal structures. The Python Materials Genomics (pymatgen) [24] package was used for all input file generation and post-processing of results.

\subsection{Diffusion energy barrier calculations for bulk undoped and doped NASICON}

Vacancy diffusion barriers in a conventional NASICON cell were calculated using the climbing-image nudged elastic band (CI-NEB) method [25]. A Monkhorst-Pack $k$-point grid of $4 \times 4 \times 2$ was used. Atomic positions were relaxed until forces were smaller than 0.02 $\mathrm{eV} / \AA \AA$. Charge neutrality, where necessary, was maintained via a compensating background charge.

\subsection{Ab initio molecular dynamics (AIMD) simulations of $\mathrm{Na}_{3} \mathrm{PO}_{4}$}

Due to the disorder in cubic $\mathrm{Na}_{3} \mathrm{PO}_{4}$, the conductivity of $\mathrm{Mg}$ doped c- $\mathrm{Na}_{3} \mathrm{PO}_{4}$ was calculated using AIMD simulations. Non-spin-polarized AIMD simulations were conducted in the NVT ensemble at $800,900,1000,1100,1200 \mathrm{~K}$. A plane-wave energy cutoff of $520 \mathrm{eV}$ and $\Gamma$ centered $1 \times 1 \times 1 k$-point mesh was adopted with 2 fs time steps. An in-house AIMD workflow software $[26,27]$ was used to automate the calculations. The diffusivity $(D)$ of $\mathrm{Na}^{+}$was calculated using the equation: 


$$
D=\frac{1}{2 d t}\left\langle[\Delta \vec{r}(t)]^{2}\right\rangle
$$

where $d$ is the dimensionality of diffusion; $[\Delta \vec{r}(t)]^{2}$ is the average mean square displacement (MSD) over a time duration $t$. The ionic conductivity, $\sigma$, is calculated using the NernstEinstein equation:

$$
\sigma=\frac{\rho z^{2} F^{2}}{R T} D_{o} e^{-\Delta E_{a} / k T}
$$

where $\rho$ is the molar density of diffusing $\mathrm{Na}$ ions in the unit cell; $z=1$ is the charge of $\mathrm{Na}$ ions; $F$ is the Faraday's constant; $\mathrm{R}$ is the gas constant and $T$ is the temperature. $D_{o}$ and $\Delta E_{a}$ are constants computed by fitting an Arrhenius relationship between diffusivities and temperatures. Room temperature diffusivity and conductivity were extrapolated from the computed quantities.

\subsection{Dopant formation energy $\left(E_{f}\right)$}

In order to determine which phase $\mathrm{Mg}$ segregates/dopes, we calculate the dopant formation energy, $E_{f}$, from first principles calculations. $E_{f}$ is a measure of the thermodynamic energy cost function required to dope a host. It is defined as:

$$
E_{f}=E_{\text {doped }}-E_{\text {pristine }}-\sum_{i=1}^{n} \mu_{i} N_{i}
$$

wherein, $E_{\text {doped }}$ is the energy of the doped chemistry, $E_{\text {pristine }}$ is the energy of the undoped chemistry and $\mu_{i}$ is the chemical potential of the dopant. In the case of $\mathrm{Mg}$ doping of NASICON, we considered two possibilities:

(a) $\mathrm{Na}_{3} \mathrm{Zr}_{2} \mathrm{Si}_{2} \mathrm{PO}_{12}+0.25 \mathrm{Mg}+0.5 \mathrm{Na} \rightarrow \mathrm{Na}_{3.5} \mathrm{Zr}_{1.75} \mathrm{Mg}_{0.25} \mathrm{Si}_{2} \mathrm{PO}_{12}+0.25 \mathrm{Zr}$

(b) $\mathrm{Na}_{3} \mathrm{PO}_{4}+0.25 \mathrm{Mg} \rightarrow \mathrm{Na}_{2.5} \mathrm{Mg}_{0.25} \mathrm{PO}_{12}+0.5 \mathrm{Na}$

wherein, Equation (a) is the doping reaction expected when $\mathrm{Mg}$ substitutes $\mathrm{Zr}$ in NASICON while Equation (b) is the doping reaction when $\mathrm{Mg}$ substitutes $\mathrm{Na}$ in $\mathrm{Na}_{3} \mathrm{PO}_{4}$. 


\section{Results}

\subsection{Synthesis and phase identification}

NASICON $\left(\mathrm{Na}_{3} \mathrm{Zr}_{2} \mathrm{Si}_{2} \mathrm{PO}_{12}\right)$ doped with different nominal atomic concentrations (0-0.9 at. \%) of $\mathrm{Mg}$ were synthesized using solid-state methods (see Methods section for details). Henceforth, all $\mathrm{Mg}$ concentrations refer to at. $\%$ of $\mathrm{Mg}$ per formula unit of $\mathrm{Na}_{3} \mathrm{Zr}_{2} \mathrm{Si}_{2} \mathrm{PO}_{12}$. It should be noted in this work, the term "doped" is used loosely to refer to NASICON synthesized with different overall atomic concentrations of $\mathrm{Mg}$ precursor added; as outlined in subsequent sections, both experimental and theoretical evidence demonstrate that the bulk NASICON crystal has limited solubility for $\mathrm{Mg}$.

Fig. 2a shows the X-ray diffraction patterns of the undoped and Mg-doped NASICONs. In agreement with previous experimental reports, $\mathrm{Na}_{3} \mathrm{PO}_{4}$ and $\mathrm{ZrO}_{2}$ phases are the main impurity phases, which were labeled by “•” and “*”, respectively, in XRD patterns of undoped and Mg-doped NASICON specimens [4]. Slow-scan XRD experiments reveal that the peak correlated with monoclinic C2/c NASICON (at about 19 degrees) is evolving even at low $\mathrm{Mg}$ dopant concentrations (Fig. 2b). The change in the shape of this peak has previously been used to study the phase transformation in NASICON from monoclinic (C2/c space group) to rhombohedral ( $\mathrm{R} \overline{3} \mathrm{c}$ space group) at high temperatures. In addition, the intensity of the peak associated with cubic $\gamma-\mathrm{Na}_{3} \mathrm{PO}_{4}$ phase (at about 21 degrees) increases with increasing nominal concentrations of $\mathrm{Mg}$ in the specimen (Fig. 2c). A phase profile fitting of the XRD pattern reveals the three main phases formed in the 0.128 at. \% $\mathrm{Mg}$-doped specimen to be $\mathrm{C} 2 / \mathrm{c}$ NASICON, $\mathrm{ZrO}_{2}$ and $\mathrm{Mg}$-doped $\gamma-\mathrm{Na}_{3} \mathrm{PO}_{4}$ (Fig. 2d). Further increase in $\mathrm{Mg}$ concentration leads to transformation of the NASICON from the monoclinic to rhombohedral phase, eventually resulting in decomposition to $\mathrm{Na}_{4} \mathrm{Zr}_{2}\left(\mathrm{SiO}_{4}\right)_{3}, \mathrm{NaMgPO}_{4}$ and $\mathrm{ZrO}_{2}$ phases in 
specimen at 0.9 at. $\%$ of $\mathrm{Mg}$. A very similar trend is also observed in XRD patterns of Nidoped NASICON specimens (Fig. S5 in Supplementary Information).
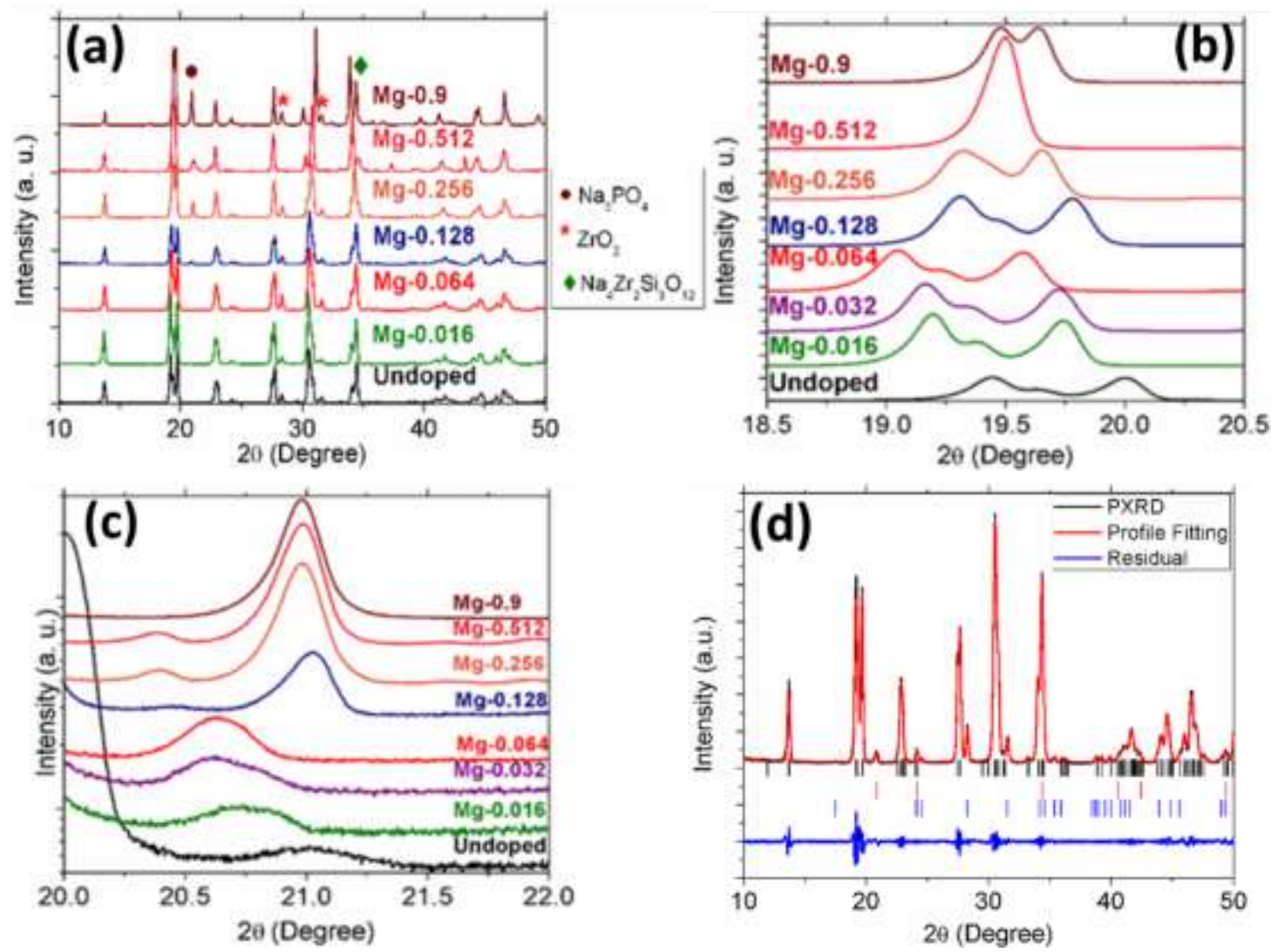

Fig. 2. Phase identification of undoped and Mg-doped NASICON. (a) XRD pattern of undoped and Mg-doped NASICON with different atomic percentages of Mg; (b) Highresolution XRD patterns showing the evolution of NASICON crystal structure from $\mathrm{C} 2 / \mathrm{c}$ (monoclinic) to $\mathrm{R} \overline{3} \mathrm{c}$ space group (rhombohedral) in Mg-doped NASICON specimens with different $\mathrm{Mg}$ concentration; (c) High-resolution XRD patterns showing the $\mathrm{Na}_{3} \mathrm{PO}_{4}$ phase peak in undoped and Mg-doped NASICON specimens with different Mg concentration; (d) Phase profile fitting of Mg-doped NASICON $\left(\mathrm{Na}_{3.256} \mathrm{Mg}_{0.128} \mathrm{Zr}_{1.872} \mathrm{Si}_{2} \mathrm{PO}_{12}\right)$ representing three main phases formed: $\mathrm{C} 2 / \mathrm{c}$ NASICON, $\mathrm{ZrO}_{2}$ and $\mathrm{Mg}$-doped $\gamma-\mathrm{Na}_{3} \mathrm{PO}_{4}$.

\subsection{Microstructure analysis}


Fig. 3 shows an SEM image of the microstructure of 0.256 at. \% Mg-doped NASICON. Three different phases with distinct contrasts are observed in the SEM images. The phase with a very bright contrast can be attributed to $\mathrm{ZrO}_{2}$ phase. EDS analysis reveals that $\mathrm{Mg}$ is not uniformly distributed in these phases and is mostly segregated in a phase depleted of Si and enriched in Na. Due to the overlap of $\mathrm{Zr} \mathrm{L}_{\alpha}(2.04 \mathrm{KeV})$ and $\mathrm{P} \mathrm{K}_{\alpha}(2.02 \mathrm{KeV})$ lines, the EDS peaks for $\mathrm{Zr}$ and $\mathrm{P}$ overlap therefore one elemental map is shown for $\mathrm{P} / \mathrm{Zr}$ elements in Fig. 3e. The non-uniform distribution of $\mathrm{Mg}$ is also observed in specimen with lower concentration of $\operatorname{Mg}(0.128$ at. \%), as shown in Fig. S6 in Supplementary Information. These observations suggest that Mg has a low solubility in the NASICON structure, and instead tends to dissolute more (preferentially "segregate") to the Si-poor secondary phase at grain boundaries. The non-uniform distribution of dopant is also observed in Ni-doped NASICON specimens (Fig. S7 in Supplementary Information).
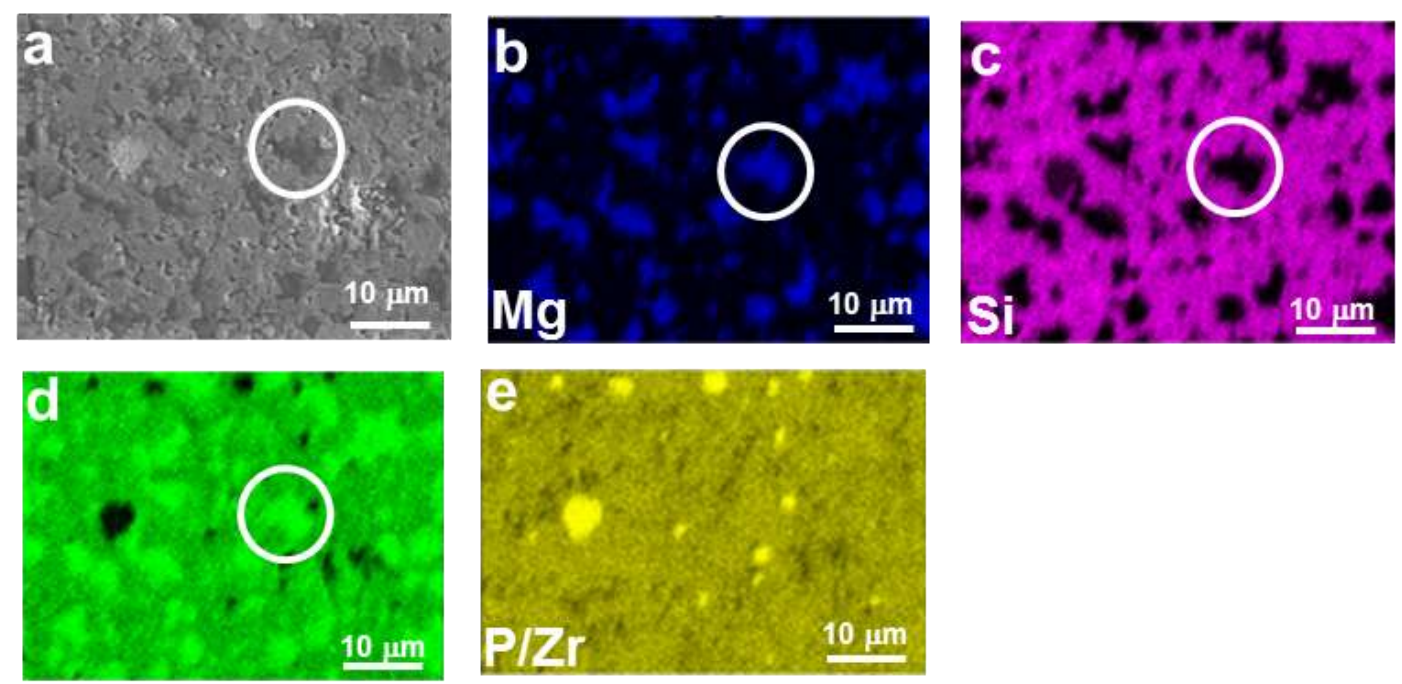

Fig. 3. (a) $\mathrm{SEM}$ image of Mg-doped NASICON $\left(\mathrm{Na}_{3.512} \mathrm{Mg}_{0.256} \mathrm{Zr}_{1.744} \mathrm{Si}_{2} \mathrm{PO}_{12}\right)$, along with individual EDS elemental maps of (b) $\mathrm{Mg}$, (c) Si, (d) $\mathrm{Na}$ and (e) P/Zr. Due to the overlap of Zr L $\mathrm{L}_{\alpha}(2.04 \mathrm{KeV})$ and $\mathrm{P} \mathrm{K}_{\alpha}(2.02 \mathrm{KeV})$ lines, one EDS elemental map is used to represent the sum of the X-ray signals from both elements. 
To confirm these observations, we calculated the dopant formation energies of $\mathrm{Mg}$ in both the bulk phase $\left(\mathrm{Na}_{3} \mathrm{Zr}_{2} \mathrm{Si}_{2} \mathrm{PO}_{12}\right)$ and the precipitation phase at grain boundaries $\left(\gamma-\mathrm{Na}_{3} \mathrm{PO}_{4}\right)$ using DFT calculations. In the bulk $\mathrm{Na}_{3} \mathrm{Zr}_{2} \mathrm{Si}_{2} \mathrm{PO}_{12}$ phase, the $\mathrm{Mg}$ dopant is found to prefer the $\mathrm{Zr}$ site with the creation of charge-compensating $\mathrm{Na}$ interstitials, with a dopant formation energy of $1.12 \mathrm{eV}$ for a $\mathrm{Mg}$ :O ratio of 0.0208 . In the secondary $\gamma-\mathrm{Na}_{3} \mathrm{PO}_{4}$ phase that forms at grain boundaries, $\mathrm{Mg}$ substitutes for $\mathrm{Na}$ with the creation of vacancies with a lower dopant formation energy of $0.85 \mathrm{eV}$ at a much higher $\mathrm{Mg}: \mathrm{O}$ ratio of 0.0625 . These DFT results suggest that $\mathrm{Mg}$ doping should preferentially dissolute (segregate) into the secondary $\gamma$ $\mathrm{Na}_{3} \mathrm{PO}_{4}$ precipitation phase at grain boundaries than the bulk $\mathrm{Na}_{3} \mathrm{Zr}_{2} \mathrm{Si}_{2} \mathrm{PO}_{12}$ phase, especially if $\mathrm{Na}$ loss during high temperature synthesis reduces the $\mathrm{Na}$ chemical potential, which would promote the formation of $\mathrm{Na}$ vacancies over $\mathrm{Na}$ interstitials.

\subsection{Ionic Conductivity}

Fig. 4a shows the total ionic conductivity of Mg-doped NASICON (0-0.9 at. \%) at room temperature $\left(25^{\circ} \mathrm{C}\right)$. The total ionic conductivity increases slightly at low concentrations of $\mathrm{Mg}$ reaching a maximum ionic conductivity of $2.05 \mathrm{mS} / \mathrm{cm}$ at 0.128 at. $\%$ of $\mathrm{Mg}$ and decreases with a further increase in the $\mathrm{Mg}$ concentration. The bulk conductivity, apparent grain boundary conductivity, and specific grain boundary conductivity determined via Brick Layer Model (BLM) are also shown in Fig. 4a. The bulk conductivity increases sharply with the increasing overall $\mathrm{Mg}$ composition below 0.064 at. \%. The conductivity only increases slightly from $0.064 \%$ to 0.128 at. $\%$ and then decreases with further increasing $\mathrm{Mg}$ doping; the decrease can be attributed to the phase transformation of NASICON crystal structure from $\mathrm{C} 2 / \mathrm{c}$ (monoclinic) to $\mathrm{R} \overline{3} \mathrm{c}$ space group (rhombohedral) in Mg-doped NASICON specimens, which is discussed in more detail later. The grain boundary conductivity, on the other hand, peaks at 0.128 at. $\% \mathrm{Mg}$, and decreases with higher $\mathrm{Mg}$ concentrations. These trends are also reflected in the bulk, grain boundary and total activation barriers for Na conduction (Fig. 4b). 
The minimums in grain boundary and total activation barriers are observed at 0.128 at. $\% \mathrm{Mg}$, and increases with higher $\mathrm{Mg}$ concentrations. We should also note that although $\mathrm{Mg}$ (and Ni) doping reduced the detrimental effects of insulating grain boundaries/secondary phases, the specific grain boundary conductivity (with the improvement due to doping) is still substantially lower than the bulk conductivity in the current case so that the total conductivity is lower than the bulk conductivity (Fig. 4(a)). Thus, a coarser microstructure should still be preferred for improving the total ionic conductivity in the current systems.

Here, we again emphasize that the so-called "grain boundary conductivity" measured by EIS in fact include contributions from grain boundaries, secondary phases, and other microstructural features, while we still call it "grain boundary" conductivity for simplicity, following the convention used in literature.

Conductivity measurements using non-blocking ( $\mathrm{Na}$ metal) electrodes was performed on $\mathrm{Mg}$ doped NASICON $\left(\mathrm{Na}_{3.256} \mathrm{Mg}_{0.128} \mathrm{Zr}_{1.872} \mathrm{Si}_{2} \mathrm{PO}_{12}\right)$ at $25{ }^{\circ} \mathrm{C}$ to verify the conductivity of $\mathrm{Mg}$ doped NASICON is ionic (see Supplementary Fig. S13). A total conductivity of about 1.9 $\mathrm{mS} / \mathrm{cm}$ was achieved, which is close to the values obtained by Pt blocking electrodes $(2.07$ $\mathrm{mS} / \mathrm{cm})$.
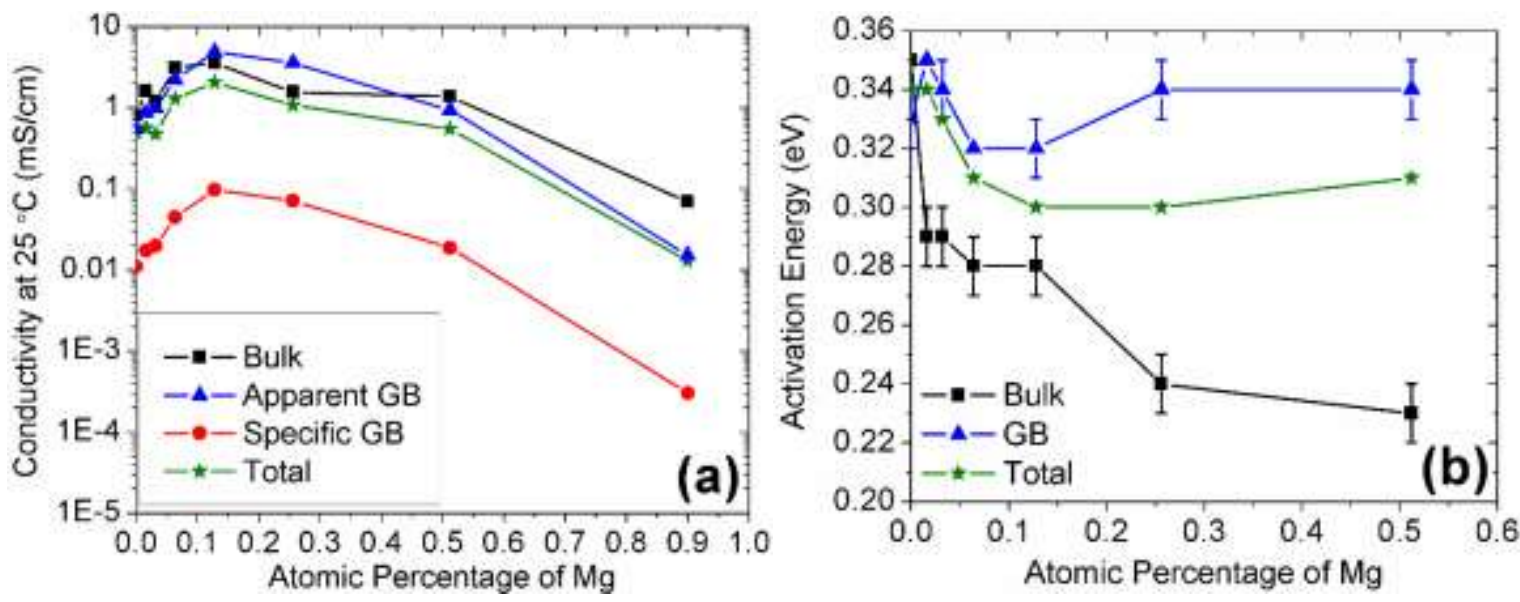
Fig. 4. (a) Bulk, apparent GB conductivity, specific GB conductivity and total ionic conductivity at room temperature $\left(25^{\circ} \mathrm{C}\right)$ for undoped and $\mathrm{Mg}$-doped NASICON $\left(\mathrm{Na}_{3} \mathrm{Zr}_{2} \mathrm{Si}_{2} \mathrm{PO}_{12}\right.$ based series). The estimated errors of conductivities are $\pm 10 \%$. (b) Activation energies of grain boundary, bulk and total conductivities for undoped and Mg-doped NASICON $\left(\mathrm{Na}_{3} \mathrm{Zr}_{2} \mathrm{Si}_{2} \mathrm{PO}_{12}\right.$ based series). Error bars are standard errors based on $95 \%$ confidence level.

\subsection{Role of Mg doping on bulk and secondary phase (grain boundary) conductivity: Hypotheses supported by AIMD modeling}

From the results in the preceding sections, we may surmise that although $\mathrm{Mg}$ does not have significant solubility in the bulk $\mathrm{Na}_{3} \mathrm{Zr}_{2} \mathrm{Si}_{2} \mathrm{PO}_{12}$ phase, it nevertheless has a significant impact on both the bulk and "grain boundary" conductivities and activation energies. These seemingly contradictory observations can be reconciled by considering the effect of $\mathrm{Mg}$ on the synthesized primary (bulk) and secondary phase equilibria. It is well-known that the NASICON structure exists in a range of compositions with formula $\mathrm{Na}_{1+x} \mathrm{Zr}_{2} \mathrm{Si}_{x} \mathrm{P}_{3-x} \mathrm{O}_{12}$ with different $\mathrm{Si} / \mathrm{P}$ ratios and correspondingly different $\mathrm{Na}$ concentrations. We therefore propose that the addition of the $\mathrm{MgO}$ precursor results in the formation of a bulk phase with a higher $\mathrm{Si} / \mathrm{P}$ ratio (and higher $\mathrm{Na}$ concentration) and a $\mathrm{Mg}$-doped $\mathrm{Na}_{3} \mathrm{PO}_{4}$ secondary phase.

To understand the role of $\mathrm{Si} / \mathrm{P}$ ratio on the activation energy, we performed climbing imagenudged elastic band calculations (CI-NEB) to determine the alkali vacancy diffusion energy barriers for monoclinic NASICON $\mathrm{Na}_{1+x} \mathrm{Zr}_{2} \mathrm{Si}_{x} \mathrm{P}_{3-x} \mathrm{O}_{12}$ for $1.75 \leq x \leq 2.25$. It has been well established that outside this range of Si concentration, NASICON chemistries have much lower ionic conductivities $[1,28,29]$. Table 1 tabulates the vacancy diffusion energy barriers for NASICON chemistries with $x=1.75,2.0$ and 2.25. At $x=2$, the maximum barrier along the percolating path (Fig. S8 in the Supplementary Information) was found to be $183 \mathrm{meV}$. 
When $\mathrm{P}$ content is increased to $1.25(x=1.75)$, the energy barrier in the local environment increases significantly to $385 \mathrm{meV}$. Conversely, when the Si content is increased to $x=2.25$, the barrier decreases slightly to $180 \mathrm{meV}$. The CI-NEB barrier plots can be found in Fig. S9 and Fig. S10 in Supplementary Information. Thus, within the monoclinic range of Si concentration, it is important that molar concentration of Si must be higher than 2.0 to achieve maximum bulk conductivity.

To investigate the effect of $\mathrm{Mg}$ doping on the conductivity of the secondary $\gamma-\mathrm{Na}_{3} \mathrm{PO}_{4}$ phase (that precipitates at grain boundaries and contributes to the "grain boundary conductivity" measured by EIS), ab initio molecular dynamics (AIMD) calculations were carried out on Mg-doped $\gamma-\mathrm{Na}_{3} \mathrm{PO}_{4}\left(\mathrm{Na}_{2.5} \mathrm{Mg}_{0.25} \mathrm{PO}_{4}\right)$ as well as t-Na $\mathrm{PO}_{4}$. Fig. 5 shows the Arrhenius relationship of diffusivity with temperature for $\gamma-\mathrm{Na}_{2.5} \mathrm{Mg}_{0.25} \mathrm{PO}_{4}$. The extrapolated bulk conductivity of $\mathrm{Na}_{2.5} \mathrm{Mg}_{0.25} \mathrm{PO}_{4}$ at room temperature $(300 \mathrm{~K})$ is $0.047 \mathrm{mS} / \mathrm{cm}$, and the predicted activation energy is $376 \mathrm{meV}$. In contrast, $\mathrm{t}-\mathrm{Na}_{3} \mathrm{PO}_{4}$ exhibits negligible diffusivity even at $800-1200 \mathrm{~K}$ in the AIMD simulations (Fig. S11 in the Supplementary Information), and its activation energy has previously been reported to be $\sim 760 \mathrm{meV}$ [30]. The modest ionic conductivity of $\mathrm{Mg}$-doped $\gamma-\mathrm{Na}_{3} \mathrm{PO}_{4}$ helps in decreasing the effective grain boundary resistance of $\mathrm{Na}$ diffusion in the $\mathrm{GB}$ as compared to the (insulating) secondary $\mathrm{ZrO}_{2}$ and $\mathrm{t}$ $\mathrm{Na}_{3} \mathrm{PO}_{4}$ phases in undoped NASICON GBs. 


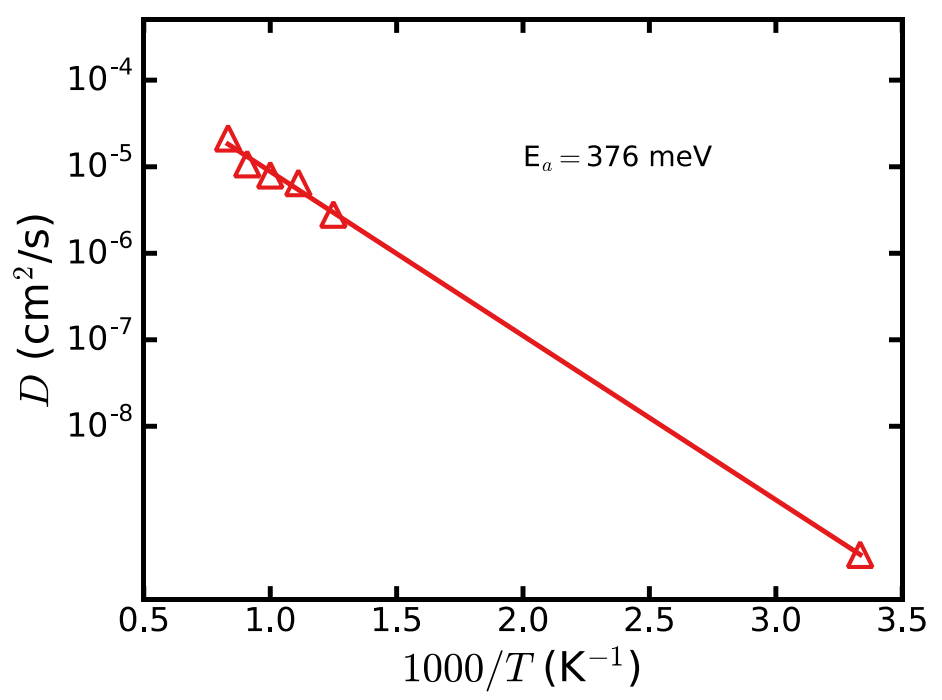

Fig. 5. Arrhenius plot of diffusivity in $\mathrm{Na}_{10} \mathrm{MgP}_{4} \mathrm{O}_{12}$ computed from ab initio molecular dynamics. Simulations were performed at 800, 900, 1000, 1100 and $1200 \mathrm{~K}$ with room temperature diffusivity being calculated via extrapolation.

\subsection{Experimental validation of hypotheses and synthesis of high-conductivity NASICON}

To further confirm the above hypotheses experimentally, monoclinic NASICON specimens with a higher nominal $\mathrm{Si} / \mathrm{P}$ ratio of 2.2/0.8 were synthesized with and without $\mathrm{Mg}$ doping. The undoped NASICON, with a nominal formula of $\mathrm{Na}_{3.2} \mathrm{Zr}_{2} \mathrm{Si}_{2.2} \mathrm{P}_{0.8} \mathrm{O}_{12}$, was found to have a bulk ionic conductivity of $5.66 \mathrm{mS} / \mathrm{cm}$, slightly higher than that of undoped $\mathrm{Na}_{3} \mathrm{Zr}_{2} \mathrm{Si}_{2} \mathrm{PO}_{12}$ with Si:P ratio of $2(5.06 \mathrm{mS} / \mathrm{cm})$. Further, the maximum total ionic conductivity achieved in $\mathrm{Mg}$ doped NASICON (Si-2.2 version: $2.7 \mathrm{mS} / \mathrm{cm}$ at 0.0625 at. $\% \mathrm{Mg}$ ) is about $30 \%$ higher than the one achieved in Mg-doped NASICON (Si-2.0 version: $2.05 \mathrm{mS} / \mathrm{cm}$ at 0.125 at. $\% \mathrm{Mg}$ ). Note that the maximum total ionic conductivity in the $\mathrm{Si}-2.2$ version is obtained at much lower concentration of $\mathrm{Mg}$ compared to the $\mathrm{Si}-2.0$ version given that even a small depletion of $\mathrm{P}$ from the bulk into the secondary $\mathrm{Na}_{3} \mathrm{PO}_{4}$ phase at grain boundaries is likely to result in a change from the monoclinic structure to the lower-conductivity rhombohedral structure [1]. Similar synthesis of Ni doped NASICON with Si:P ratio of 2.2/0.8 was found to achieve a maximum ionic conductivity of $2.3 \mathrm{mS} / \mathrm{cm}$, as shown in Fig. 6 . 


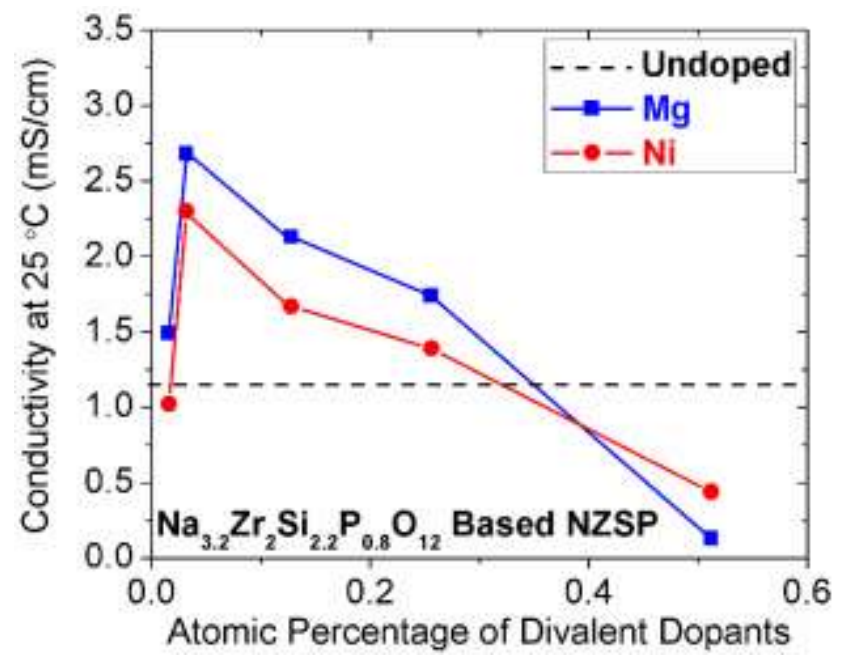

Fig. 6. Total ionic conductivity at room temperature $\left(25^{\circ} \mathrm{C}\right)$ of undoped and doped $(\mathrm{Mg} / \mathrm{Ni})$ NASICON $\left(\mathrm{Na}_{3.2} \mathrm{Zr}_{2} \mathrm{Si}_{2.2} \mathrm{P}_{0.8} \mathrm{O}_{12}\right)$ with different atomic percentages of $\mathrm{Mg} / \mathrm{Ni}$.

\section{Discussion}

In this work, we demonstrated the synthesis of a high-conductivity monoclinic $\mathrm{C} 2 / \mathrm{c}$ NASICON via the introduction of aliovalent dopants such as $\mathrm{Mg}^{2+}$ and $\mathrm{Ni}^{2+}$. We present clear experimental and computational evidence that the effect of aliovalent dopants are more complex than that has been previously assumed. The commonly held assumption in previous works is that any dopants introduced would sit in either the $\mathrm{Zr}^{4+}$ or $\mathrm{Na}^{+}$sites, resulting in a modification of the bulk activation barriers and/or $\mathrm{Na}^{+}$concentration. Our EDS results and calculated dopant formation energies suggest that dopants are more likely to incorporate into a secondary phase precipitated at grain boundaries, such as $\mathrm{Na}_{3} \mathrm{PO}_{4}$, and any effect on the bulk activation barriers result from changes in the Si:P ratio within the bulk. Indeed, CI-NEB calculations confirm the lowering of the bulk activation barriers with increase in $\mathrm{Si} / \mathrm{P}$ ratio, subject to the restriction that $\mathrm{Si} / \mathrm{P}$ must be less than 2.2 to prevent transformation to the lowerconducting rhombohedral phase. This is consistent with previous experimental results by Ahmad et al. reporting maximum room temperature bulk ionic conductivity for the 
NASICON for a Si/P ratio of 2.2/0.8 [28]. On the other hand, CI-NEB calculations performed on Mg-doped NASICON (where Mg is assumed to sit in the $\mathrm{Zr}$ site) find that bulk activation barriers in the vicinity of the dopant increases significantly from $183 \mathrm{meV}$ to $407 \mathrm{meV}$ (see Fig. S12 (a) in Supplementary Information), directly contradicting previously held assumptions that aliovalent dopants decrease the bulk activation energies. Similarly, CI-NEB calculations performed on Ni-doped NASICON also shows that the activation energy increases to $458 \mathrm{meV}$ in the vicinity of the dopant (See Fig. S12 (b) in Supplementary Information).

Though the introduction of aliovalent dopants does have an indirect effect on the bulk conductivity by modifying the $\mathrm{Si} / \mathrm{P}$ ratio, it has a far more important effect on the effective grain boundary conductivity. Like many oxide superionic conductors, the bulk conductivity is not the limiting bottleneck in the NASICON phases, and bulk conductivities in excess of 1 $\mathrm{mS} / \mathrm{cm}$ have been reported by Ahmad et al. since 1987 [28]. The EIS results in this work (Fig. 4) clearly show a substantial improvement in the effective grain boundary conductivity upon doping, which is validated by AIMD simulations showing significant improvement in the conductivity of the secondary phase at grain boundaries. Indeed, it is our belief that similar effects are present in previous reports of enhanced total conductivity in NASICON with other dopants such as $\mathrm{Sc}^{3+}$ and $\mathrm{Y}^{3+}[4,22,31]$. Table $\mathrm{S} 1$ in Supplementary Information shows the dopant formation energies in the bulk NASICON and the predicted secondary phase (that presumably precipitates at grain boundaries) for these dopants; similarly, a higher dopant solubility is predicted for the secondary phase compared to the primary bulk phases.

It should be noted that during the course of this manuscript preparation, a very recent report of Mg-doped NASICON published, claiming a conductivity of $3.5 \mathrm{mS} / \mathrm{cm}$ at room temperature for a low doping level of 0.05 at. $\% \mathrm{Mg}$ [32]; however, our measurements showed that 
specimens with a similar composition $(0.064$ at. $\% \mathrm{Mg})$ only have conductivity of $\sim 1.3$ $\mathrm{mS} / \mathrm{cm}$, which we believe to be a more reasonable value since at these low doping concentrations, the nominal concentration of $\mathrm{Na}$ is lower than undoped NASICON (Si-2.2 version). In addition, the conductivity of undoped NASICON is not reported in that study, making it difficult to assess the actual improvement upon doping and effects of other factors. We also notice an unusual high contact resistance $(120 \Omega)$ in the Nyquist plot in that report [32], which should have been deducted from total resistivity to calculate the conductivity of Mg-doped NASICON, but may cause some measurement errors. Moreover, authors attributed the improvement in conductivity to the increased bottleneck size (being related to a pseudohexagonal ring consisting of alternating three $\mathrm{ZrO}_{6}$ octahedra and three $\mathrm{SiO}_{4} / \mathrm{PO}_{4}$ tetrahedra) for ion conduction, which is likely an overly-simplified explanation. While isovalent doping can be purely attributed to structural changes [33], it is generally believed that the effects of aliovalent doping on conductivity involves complex interactions of an increased concentration of mobile species, as well as modified electronic density and framework structure, albeit the additional (yet important) effects on solubility, phase transformation, and secondary phase discovered in this study. For example, a recent study of Al-doped $\mathrm{LiTi}_{2}\left(\mathrm{PO}_{4}\right)_{3}[34$ ] has shown that the activation energy along the diffusion pathway actually increases in the vicinity of the dopant. Our CI-NEB calculations performed on $\mathrm{Mg} / \mathrm{Ni}$-doped NASICON (where $\mathrm{Mg} / \mathrm{Ni}$ is assumed to sit in the $\mathrm{Zr}$ site) also finds that bulk activation barriers in the vicinity of the dopant increases significantly as discussed above. Similar to previous reports [5], a high GB conductivity was also reported, for which the authors did not provide any explanation [32].

Separating the bulk and GB conductivities, we have shown that the effective GB conductivity of Mg-doped NASICON is also improved in comparison with undoped NASICON. Such improvements in the GB conductivity of aliovalent-doped NASICON has been observed previously (in the normalized Nyquist plots in Fig. 1a and b in reference 4 and table 2 in 
reference 5); but, unfortunately, these observations have not been fully recognized and elaborated in previous studies [4,5]. Moreover, adding $\mathrm{ZnO}$ as a sintering aid to $\mathrm{NaZr}_{2}\left(\mathrm{PO}_{4}\right)_{3}$ was proposed to improve the density of ceramic by creating positively charged oxygen vacancies to maintain charge neutrality with negatively charged zinc substituted on $\mathrm{Zr}$ sites [35]. Here, it was suggested that oxygen vacancies may reduce the structural perfection of sodium zirconium phosphate to improve the sintering behavior in presence of zinc oxide [35]. However, in our cases, the densities of sintered specimens with different atomic concentrations of divalent dopants were all about $90 \%$ of theoretical density; thus, the improvements in grain boundary conductivity cannot be simply attributed to improved densities. In addition, our EDS results and calculated dopant formation energies suggest that dopants are more likely to be incorporated in a secondary phase at grain boundaries, such as $\mathrm{Na}_{3} \mathrm{PO}_{4}$. The high GB resistivity of NASICON is mostly attributed to the secondary $\mathrm{ZrO}_{2}$ phase precipitated at GBs due to evaporation of $\mathrm{Na}$ and $\mathrm{P}$ during high temperature synthesis and sintering of NASICON [18-20]. However, the $\mathrm{ZrO}_{2}$ phase is also reported to form in NASICON sintered by field assisted sintering technique (FAST) with very short exposure to high temperatures [36]. It is difficult to separate out the grain boundary and bulk conductivity at high temperatures for superionic conductors due to electrode effects and inductions from connections and measurement cell [37]. A similar behavior, i.e., a very low resistivity of grain boundaries at high temperatures and high resistivity at intermediate and low temperatures, was reported for NASICON $[18,29]$. $\mathrm{Na}_{3} \mathrm{PO}_{4}$ is another phase that often precipitate at grain boundaries of NASICON. Its cubic phase ( $\gamma$ phase) stabilized at high temperatures is considered as solid electrolyte for high temperature applications ( $\mathrm{Na}-\mathrm{S}$ batteries) [30,38-40], while its low-temperature phase (tetragonal $\mathrm{Na}_{3} \mathrm{PO}_{4}$ ) has an extremely low ionic conductivity at intermediate and low temperatures. The low conductivity is attributed to the compact crystal structure of $\mathrm{Na}_{3} \mathrm{PO}_{4}$ and low concentrations of $\mathrm{Na}$ vacancies. As shown in this work, aliovalent dopants such as $\mathrm{Mg}^{2+}$ tend to displace $\mathrm{Na}$ in the secondary $\mathrm{Na}_{3} \mathrm{PO}_{4}$ phase at grain 
boundaries, creating $\mathrm{Na}$ vacancies. We further speculate that this $\mathrm{Na}$ displacement from the GB phase results in the injection of Na into the vacant sites in the NASICON crystal structure. This is somewhat analogous to previously investigated mixed-phase ionic conductors show a higher ionic conductivity than those of the parent phases in $\mathrm{Ag}^{+}$ionic conductors $[41,42]$ and in $\mathrm{F}^{-}$ion conductors [43-45]. It is possible that a unilateral distribution of charge carrier $\left(\mathrm{Na}^{+}\right)$ from $\mathrm{Mg}$-doped $\mathrm{Na}_{3} \mathrm{PO}_{4}$ phase into vacant sites in NASICON structure that improves the ionic conductivity of NASICON. Comparing normalized Nyquist plots at $25{ }^{\circ} \mathrm{C}$ for $\mathrm{Mg} 0.128$ at. \% and $\mathrm{Mg} 0.256$ at. \% specimens (Fig. S1 in Supplementary Information), it is obvious that both bulk and grain boundary conductivity decreases at higher concentrations due to coarsening of $\mathrm{Mg}$-doped $\mathrm{Na}_{3} \mathrm{PO}_{4}$ phase. This is presumably due to 1) a depletion of $\mathrm{P}$ in the primary NASICON bulk phase and formation of R $\overline{3} \mathrm{c}$ NASICON that decreases the bulk conductivity and 2) a decrease in volume fraction of NASICON/Mg-doped $\mathrm{Na}_{3} \mathrm{PO}_{4}$ interface. The conductivity drop at high concentrations of aliovalent-doped NASICON $\left(\mathrm{Sc}^{3+}\right.$-doped NASICON) [5] was previously attributed to decrease in $\mathrm{Na}$ vacant sites in NASICON structure; we speculate that the maximum conductivity achieved in Mg-doped NASICON is limited by size and distribution of secondary $\mathrm{Mg}$-doped $\mathrm{Na}_{3} \mathrm{PO}_{4}$ phase at grain boundaries in NASICON, following to a percolation theory for composite ionic conductors.

It is also possible that a $\mathrm{Na}_{3} \mathrm{PO}_{4}$-based, nanoscale, interfacial phase formed in doped NASICON materials, serving as an additional reason for improved effective grain boundary conductivity. A similar case has been reported previously, where the formation of 1-4 nmthick, $\mathrm{LaP}_{3} \mathrm{O}_{9}$-based, interfacial phases at grain boundaries in $\mathrm{LaPO}_{4}$ increased the proton conductivity by more than 10 times [46]. Moreover, the formation of $\mathrm{Li}_{4} \mathrm{P}_{2} \mathrm{O}_{7^{-}}$or $\mathrm{Li}_{3} \mathrm{PO}_{4}-$ based nanoscale surface phases have been shown to improve the rate capabilities of Li-ion batteries [47,48], pseudocapacitors [49] and a similar surface phase was recently found to improve the oxygen ionic conductivity of nanofibers by more than 1000 times [50], see a 
recent review for relevant discussion [51]. Unfortunately, these NASICON materials are extremely sensitive to electron and ion beams, preventing us from conducting direct atomicresolution characterization of grain boundaries (as the MRSICON specimens, particularly their grain boundaries, can be damaged by the high-energy electron beam in TEM or even in high-magnification SEM). Thus, it is extremely challenging, if not infeasible, to confirm whether a similar $\mathrm{Na}_{3} \mathrm{PO}_{4}$-based, nanoscale interfacial phase formed and played a role in improving the grain boundary ionic conductivity directly through HRTEM in the current case and further discussion is thus unwarranted at this time.

Finally, this work suggested that there are significant opportunities to improve both bulk and grain boundary conductivity of solid electrolytes via controlling the microstructure (e.g., the size and distribution of the secondary phase(s) precipitated at grain boundaries) as well as interfacial engineering. Moreover, prior studies have been successfully taken advantages of two-dimensional heterostructures (that is beneficial if and only if the interfacial ionic conductivity is higher the bulk conductivity) [52] or textured columnar grains (that may minimize the detrimental grain boundary blocking effects if the grain boundary conductivity is significantly lower than the bulk conductivity) [53] to achieve much improved conductivities along one direction. Specific for this case, we expect that well-controlled microstructures and further interfacial engineering, e.g., via forming conductive nanoscale (thermodynamically 2-D) interfacial phase (complexion) [51], may help to achieve even higher $\mathrm{Na}$ ionic conductivity for doped NASICON. The assembly of this and early studies collectively suggest the important opportunities to achieve superior ionic conductivities via controlling and tailoring microstructure and interfaces, in addition to the phase and doping of the primary bulk phases.

\section{Conclusion}


To summarize, we have studied the effects of aliovalent doping on both bulk and GB conductivity of NASICON using both experimental and computational techniques. Based on these studies, Mg and Ni doped NASICONs with the optimized total ionic conductivities of $2.7 \mathrm{mS} / \mathrm{cm}$ and $2.1 \mathrm{mS} / \mathrm{cm}$, respectively, were synthesized. Our results show that higher concentrations of the $\mathrm{Mg}$ or $\mathrm{Ni}$ dopant promote the formation of a $\mathrm{Na}_{3} \mathrm{PO}_{4}$-based secondary phase. This simultaneously leads to a change in the $\mathrm{Si} / \mathrm{P}$ composition in the bulk phase, which increases the bulk ionic conductivity initially (but eventually ending up with lower conducting $\mathrm{R} \overline{3} \mathrm{c}$ phase NASICON with high doping level). More importantly, the dopant containing $\mathrm{Na}_{3} \mathrm{PO}_{4}$-based secondary phase formed at grain boundaries reduces the effective grain boundary resistance, thereby increasing the total ionic conductivity. This study suggests significant opportunities to improve the total conductivity via controlling the phase and chemistry of both the primary and secondary phases via doping, as well as tailoring the interfaces and microstructure.

\section{Acknowledgement}

We gratefully acknowledge the financial support from the National Science Foundation under a DMREF grant no. CMMI-1436976.

M.S. and B.R. contributed equally to this work. Correspondence should be addressed to: J.L. (jluo@alum.mit.edu) and S.P.O. (ongsp@eng.ucsd.edu). 
Table:

Table 1. Climbing Image Nudged Elastic Band (CI-NEB) calculation of vacancy diffusion barriers in NAICON chemistries. Si/P compositions of 1.75/1.25 and 2.25/0.75 were constructed from the structure of $\mathrm{Si} / \mathrm{P}: 2.0 / 1.0$ by substituting one $\mathrm{Si} / \mathrm{P}$ in $\mathrm{Na}_{12} \mathrm{Zr}_{8} \mathrm{Si}_{8}\left(\mathrm{PO}_{12}\right)_{4}$ accompanied by the addition of vacancy/ $\mathrm{Na}^{+}$appropriately. In the case of $\mathrm{Si} / \mathrm{P}: 2.25 / 0.75$ and $\mathrm{Si} / \mathrm{P}: 1.75 / 1.25$, the barriers were computed in the vicinity of the substituted $\mathrm{Si} / \mathrm{P}$ as compared to the nominal composition of $\mathrm{Si} / \mathrm{P}: 2.0 / 1.0$. It is assumed that the other paths in the framework are not affected by the introduction of $\mathrm{Si} / \mathrm{P}$ and the accompanying $\mathrm{Na}+/$ vacancy respectively.

\begin{tabular}{|c|c|c|c|}
\hline Si/P Composition & Si/P: $1.75 / 1.25$ & Si/P: $2.0 / 1.0$ & Si/P: $2.25 / 0.75$ \\
\hline $\begin{array}{c}\text { CI-NEB Barrier } \\
(\mathrm{meV})\end{array}$ & 385 & 183 & 180 \\
\hline
\end{tabular}




\section{References:}

[1] J.B. Goodenough, H.Y.P. Hong, J.A. Kafalas, Fast Na+-Ion Transport in Skeleton Structures, Mater. Res. Bull. 11 (1976) 203-220. doi:Doi 10.1016/0025$5408(76) 90077-5$.

[2] H.Y.P. Hong, Crystal-Structures and Crystal-Chemistry in System Na1+Xzr2sixp3Xo12, Mater. Res. Bull. 11 (1976) 173-182. doi:Doi 10.1016/0025-5408(76)90073-8.

[3] L. Vijayan, G. Govindaraj, Structural and electrical properties of high-energy ballmilled NASICON type Li1.3Ti1.7A10.3(PO4) 2.9(VO4)0.1 ceramics, J. Phys. Chem. Solids. 72 (2011) 613-619. doi:10.1016/j.jpcs.2011.02.001.

[4] A.G. Jolley, G. Cohn, G.T. Hitz, E.D. Wachsman, Improving the ionic conductivity of NASICON through aliovalent cation substitution of Na3Zr2Si2PO12, Ionics (Kiel). 21 (2015) 3031-3038. doi:10.1007/s11581-015-1498-8.

[5] Q. Ma, M. Guin, S. Naqash, C.-L. Tsai, F. Tietz, O. Guillon, Scandium-Substituted Na3Zr2(SiO4)2(PO4) Prepared by a Solution-Assisted Solid-State Reaction Method as Sodium-Ion Conductors, Chem. Mater. 0 (n.d.) null. doi:10.1021/acs.chemmater.6b02059.

[6] T. Salkus, A. Dindune, Z. Kanepe, J. Ronis, A. Urcinskas, A. Kezionis, A.F. Orliukas, Lithium ion conductors in the system $\mathrm{Li}(1+\mathrm{y}) \mathrm{Ge}(2-\mathrm{x}-\mathrm{y}) \mathrm{Ti}(\mathrm{x}) \mathrm{Aly}(\mathrm{PO})(3)(\mathrm{x}=0.1$ divided by $0.3, y=0.07$ divided by 0.21 ), Solid State Ionics. 178 (2007) 1282-1287.

[7] M. Jansen, U. Henseler, Synthesis, Structure Determination, and Ionic-Conductivity of Sodium Tetrathiophosphate, J. Solid State Chem. 99 (1992) 110-119. doi:Doi $10.1016 / 0022-4596(92) 90295-7$.

[8] A. Hayashi, K. Noi, N. Tanibata, M. Nagao, M. Tatsumisago, High sodium ion conductivity of glass ceramic electrolytes with cubic Na3PS4, J. Power Sources. 258 (2014) 420-423. doi:10.1016/j.jpowsour.2014.02.054. 
[9] A. Hayashi, K. Noi, A. Sakuda, M. Tatsumisago, Superionic glass-ceramic electrolytes for room-temperature rechargeable sodium batteries, Nat. Commun. 3 (2012). doi:Artn 85610.1038/Ncomms 1843.

[10] W.D. Richards, T. Tsujimura, L.J. Miara, Y. Wang, J.C. Kim, S.P. Ong, I. Uechi, N. Suzuki, G. Ceder, - Design and synthesis of the superionic conductor Na10SnP2S12, 7 (2016).

[11] W.D. Richards, T. Tsujimura, L. Miara, Y. Wang, J.C. Kim, S.P. Ong, I. Uechi, N. Suzuki, G. Ceder, Design and synthesis of the superionic conductor Na10SnP2S12, Nat. Commun. 7 (2016) 1-8. doi:10.1038/ncomms 11009.

[12] G. Sahu, C. Liang, Crystalline Sulfide Electrolytes for Li-S Batteries, in: Handb. Solid State Batter., n.d.: pp. 365-389. doi:10.1142/9789814651905_0011.

[13] F. Mauvy, E. Siebert, P. Fabry, Reactivity of \{NASICON $\}$ with water and interpretation of the detection limit of a $\{\mathrm{NASICON}\}$ based $\mathrm{Na}+$ ion selective electrode, Talanta. 48 (1999) 293-303. doi:http://dx.doi.org/10.1016/S00399140(98)00234-3.

[14] R.O. Fuentes, F. Figueiredo, F.M.B. Marques, J.I. Franco, Reaction of NASICON with water, Solid State Ionics. 139 (2001) 309-314. doi:10.1016/S0167-2738(01)00683-X.

[15] B. Radhakrishnan, S.P. Ong, Aqueous stability of alkali superionic conductors from first principles calculations, Front. Energy Res. 4 (2016) 1-12. doi:10.3389/fenrg.2016.00016.

[16] W. Bogusz, F. Krok, W. Jakubowski, Bulk and grain boundary electrical conductivities of NASICON, Solid State Ionics. 2 (1981) 171-174. doi:10.1016/01672738(81)90175-2.

[17] O. Bohnke, S. Ronchetti, D. Mazza, Conductivity measurements on nasicon and nasicon-modified materials, Solid State Ionics. 122 (1999) 127-136. doi:10.1016/S0167-2738(99)00062-4. 
[18] W. Bogusz, F. Krok, W. Jakubowski, Bulk and grain boundary electrical conductivities of NASICON, Solid State Ionics. 2 (1981) 171-174. doi:10.1016/01672738(81)90175-2.

[19] R. Fuentes, F.M. Figueiredo, F.M.B. Marques, J.I. Franco, Influence of microstructure on the electrical properties of NASICON materials, Solid State Ionics. 140 (2001) 173179. doi:10.1016/S0167-2738(01)00701-9.

[20] Z.-Z. ZHANG, S.-Q. SHI, Y.-S. HU, L.-Q. CHEN, Sol-Gel Synthesis and Conductivity Properties of Sodium Ion Solid State Electrolytes Na3Zr2Si2PO12, J. Inorg. Mater. 28 (2013) 1255-1260. doi:10.3724/SP.J.1077.2013.13120.

[21] A.G. Jolley, D.D. Taylor, N.J. Schreiber, E.D. Wachsman, Structural Investigation of Monoclinic-Rhombohedral Phase Transition in Na3Zr2Si2PO12 and Doped NASICON, J. Am. Ceram. Soc. 98 (2015) 2902-2907. doi:10.1111/jace.13692.

[22] W.G. Coors, J.H. Gordon, S.G. Menzer, Electrochemical cell comprising ionically conductive membrane and porous multiphase electrode, (2012). http://www.google.com/patents/US8338038.

[23] G. Kresse, J. Furthmüller, Efficient iterative schemes for ab initio total-energy calculations using a plane-wave basis set., Phys. Rev. B. Condens. Matter. 54 (1996) 11169-11186. doi:10.1103/PhysRevB.54.11169.

[24] S.P. Ong, W.D. Richards, A. Jain, G. Hautier, M. Kocher, S. Cholia, D. Gunter, V.L. Chevrier, K. a. Persson, G. Ceder, Python Materials Genomics (pymatgen): A robust, open-source python library for materials analysis, Comput. Mater. Sci. 68 (2013) 314319. doi:10.1016/j.commatsci.2012.10.028.

[25] G. Henkelman, B.P. Uberuaga, H. Jónsson, A climbing image nudged elastic band method for finding saddle points and minimum energy paths, J. Chem. Phys. 113 (2000) 9901. doi:10.1063/1.1329672.

[26] A. Jain, S.P. Ong, W. Chen, B. Medasani, X. Qu, M. Kocher, M. Brafman, G. Petretto, 
G.-M. Rignanese, G. Hautier, D. Gunter, K. a. Persson, FireWorks: a dynamic workflow system designed for highthroughput applications, Concurr. Comput. Pract. Exp. 27 (2015) 5037-5059. doi:10.1002/cpe.3505.

[27] Z. Deng, B. Radhakrishnan, S.P. Ong, Rational Composition Optimization of the Lithium-Rich Li 3 OCl 1- x Br x Anti-Perovskite Superionic Conductors, Chem. Mater. 27 (2015) 3749-3755. doi:10.1021/acs.chemmater.5b00988.

[28] A. Ahmad, T.A. Wheat, A.K. Kuriakose, J.D. Canaday, A.G. McDonald, Dependence of the Properties of NASICONs on their Composition and Processing, Solid State Ionics. 24 (1987) 89-97. doi:10.1016/0167-2738(87)90070-1.

[29] O. Bohnke, S. Ronchetti, D. Mazza, Conductivity measurements on nasicon and nasicon-modified materials, Solid State Ionics. 122 (1999) 127-136. doi:10.1016/S0167-2738(99)00062-4.

[30] J.T.S. Irvine, A.R. West, Solid electrolytes based on Na3PO4:M2+ (M=Mg, Zn, Ca, Sr), Solid State Ionics. 37 (1989) 73-78. doi:http://dx.doi.org/10.1016/01672738(89)90290-7.

[31] R.E. Clausing, F. Waelbroeck, J. Winter, P. Wienhold, L. Kiinen, N. Noda, Surface Structure Determination by X-Ray Diffraction R . Feidenhans ' L 0167-5729 / 89 / \{\$\} 29. $40\{$ \{C\} Elsevier Science Publishers B . V . ( North-Holland Physics Publishing Division ), Surf. Sci. Rep. 10 (1989) 105-188.

[32] S. Song, H.M. Duong, A.M. Korsunsky, N. Hu, L. Lu, A Na+ Superionic Conductor for Room-Temperature Sodium Batteries, Sci. Rep. 6 (2016) 32330. http://dx.doi.org/10.1038/srep32330.

[33] E.R. Losilla, M. a. G. Aranda, S. Bruque, M. a. París, J. Sanz, A.R. West, Understanding Na Mobility in NASICON Materials: A Rietveld, $23 \mathrm{Na}$ and 31 P MAS NMR, and Impedance Study, Chem. Mater. 10 (1998) 665-673. doi:10.1021/cm970648j. 
[34] B. Lang, B. Ziebarth, C. Elsässer, Lithium Ion Conduction in LiTi 2 (PO 4 ) 3 and Related Compounds Based on the NASICON Structure: A First-Principles Study, Chem. Mater. 27 (2015) 5040-5048. doi:10.1021/acs.chemmater.5b01582.

[35] M. V Sukhanov, V.I. Pet'kov, D. V Firsov, Sintering mechanism for high-density NZP ceramics, Inorg. Mater. 47 (2011) 674-678. doi:10.1134/S0020168511060197.

[36] J.-S. Lee, C.-M. Chang, Y.I.L. Lee, J.-H. Lee, S.-H. Hong, Spark Plasma Sintering (SPS) of NASICON Ceramics, J. Am. Ceram. Soc. 87 (2004) 305-307. doi:10.1111/j.1551-2916.2004.00305.x.

[37] L. Zhang, F. Liu, K. Brinkman, K.L. Reifsnider, A. V Virkar, A study of gadoliniadoped ceria electrolyte by electrochemical impedance spectroscopy, J. Power Sources. 247 (2014) 947-960. doi:http://dx.doi.org/10.1016/j.jpowsour.2013.09.036.

[38] J.T.S. Irvine, A.R. West, Solid electrolytes based on Na3PO4 doped with S, Se, Mo, W, Mater. Res. Bull. 22 (1987) 1047-1054. doi:http://dx.doi.org/10.1016/00255408(87)90233-9.

[39] W.-G. Yin, J. Liu, C.-G. Duan, W.N. Mei, R.W. Smith, J.R. Hardy, Superionicity in \$ $\backslash$ mathrm $\{\mathrm{Na}\}\}_{-}\{3\}\{\backslash \text { mathrm }\{\mathrm{PO}\}\}_{-}\{4\} \$:$ A molecular dynamics simulation, Phys. Rev. B. 70 (2004) 64302. doi:10.1103/PhysRevB.70.064302.

[40] A. Hooper, P. McGeehin, K.T. Harrison, B.C. Tofield, Ionic conductivity of pure and doped Na3PO4, J. Solid State Chem. 24 (1978) 265-275.

doi:http://dx.doi.org/10.1016/0022-4596(78)90018-X.

[41] K. Shahi, J.B. Wagner, Anomalous ionic conduction in AgBr般AgI mixed crystals and multiphase systems, J. Phys. Chem. Solids. 43 (1982) 713-722.

doi:http://dx.doi.org/10.1016/0022-3697(82)90236-0.

[42] K. Shahi, J.B. Wagner, Fast ion transport in silver halide solid solutions and multiphase systems, Appl. Phys. Lett. 37 (1980).

[43] N. Sata, N.Y. Jin-Phillipp, K. Eberl, J. Maier, Enhanced ionic conductivity and 
mesoscopic size effects in heterostructures of BaF2 and CaF2, Solid State Ionics. 154155 (2002) 497-502. doi:http://dx.doi.org/10.1016/S0167-2738(02)00488-5.

[44] N. Sata, K. Eberman, K. Eberl, J. Maier, Mesoscopic fast ion conduction in nanometrescale planar heterostructures, Nature. 408 (2000) 946-949.

http://dx.doi.org/10.1038/35050047.

[45] N.I. Sorokin, I.I. Buchinskaya, P.P. Fedorov, B.P. Sobolev, Electrical conductivity of a CaF2-BaF2 nanocomposite, Inorg. Mater. 44 (2008) 189-192. doi:10.1134/S0020168508020192.

[46] G. Harley, R. Yu, L.C. De Jonghe, Proton transport paths in lanthanum phosphate electrolytes, Solid State Ionics. 178 (2007) 769-773. doi:http://dx.doi.org/10.1016/j.ssi.2007.03.011.

[47] B. Kang, G. Ceder, Battery materials for ultrafast charging and discharging, Nature. 458 (2009) 190-193. http://dx.doi.org/10.1038/nature07853.

[48] J. Huang, J. Luo, A facile and generic method to improve cathode materials for lithium-ion batteries via utilizing nanoscale surface amorphous films of self-regulating thickness, Phys. Chem. Chem. Phys. 16 (2014) 7786-7798. doi:10.1039/C4CP00869C.

[49] M. Samiee, J. Luo, Pseudocapacitive Properties of Two-Dimensional Surface Vanadia Phases Formed Spontaneously on Titania, ACS Appl. Mater. Interfaces. 8 (2016) 12871-12880. doi:10.1021/acsami.6b03569.

[50] W. Liu, W. Pan, J. Luo, A. Godfrey, G. Ou, H. Wu, W. Zhang, Suppressed phase transition and giant ionic conductivity in La2Mo2O9 nanowires, Nat. Commun. 6 (2015) 8354. http://dx.doi.org/10.1038/ncomms9354.

[51] J. Luo, Interfacial engineering of solid electrolytes, J. Mater. 1 (2015) 22-32. doi:10.1016/j.jmat.2015.03.002.

[52] J. Maier, Nanoionics: ion transport and electrochemical storage in confined systems, Nat Mater. 4 (2005) 805-815. http://dx.doi.org/10.1038/nmat1513. 
[53] D. Pergolesi, E. Fabbri, A. D’Epifanio, E. Di Bartolomeo, A. Tebano, S. Sanna, S. Licoccia, G. Balestrino, E. Traversa, High proton conduction in grain-boundary-free yttrium-doped barium zirconate films grown by pulsed laser deposition, Nat Mater. 9 (2010) 846-852. http://dx.doi.org/10.1038/nmat2837. 